

\title{
THE CHEMICAL COMPOSITION OF COOL STARS: II - THE HYDROGEN DEFICIENT STARS
}

\author{
David L. Lambert \\ Department of Astronomy \\ The University of Texas \\ Austin, TX 78712 USA
}

\begin{abstract}
The chemical composition of the $\mathrm{R}$ Coronae Borealis and cool hydrogen deficient carbon stars is reviewed. Similarities and differences between these stars and the hot He stars are noted. Proposed origins for the hydrogen deficient stars are sketched. Recent claims that normal (spectral type N) cool carbon stars are hydrogen deficient are shown to be unfounded. Attention is drawn to the curious case of pop. II variables (RV Tauri, W Virginis, and RR Lyrae stars) whose atmospheres show striking deficiencies of heavy elements and may be hydrogen deficient.
\end{abstract}

\section{Introduction}

In a Universe in which hydrogen is so supremely abundant, the few stars with atmospheres deficient in hydrogen deserve careful study. Their compositions should offer clues to the events leading to the birth of a hydrogen deficient star. In this brief review, I discuss the cool hydrogen deficient giant and supergiant (Hd) stars. The R Coronae Borealis $(\mathrm{RCrB})$ stars define the high temperature limit of my sample.

Investigators of Hd stars hope to obtain clues to the origin of the H deficiency and to the identity of the stars' progenitors and descendants. Processes capable of transforming a star with an atmosphere of normal composition to a Hd star include severe mass loss via a stellar wind or an explosion, Roche-lobe transfer of mass across a binary system, and deep prolonged mixing leading to hydrogen burning at the base of the convective envelope. Throughout this review, I ignore the possibility that diffusion may create a hydrogen deficient atmosphere around a star with a normal (integrated) composition; my definition of the adjective 'cool' does not embrace the chemically peculiar stars of the upper main sequence.

The review begins with a full discussion of the two well-known classes of $\mathrm{Hd}$ stars - the RCrBs and the hydrogen-deficient carbon ( $\mathrm{HdC}$ ) stars. Then, I comment on claims that the atmosphere of the typical (spectral type $\mathrm{N}$ ) cool carbon star is also significantly deficient in hydrogen; I show that there is no compelling evidence for these claims. I conclude the review by highlighting several varieties of peculiar red giants which may be hydrogen deficient. 


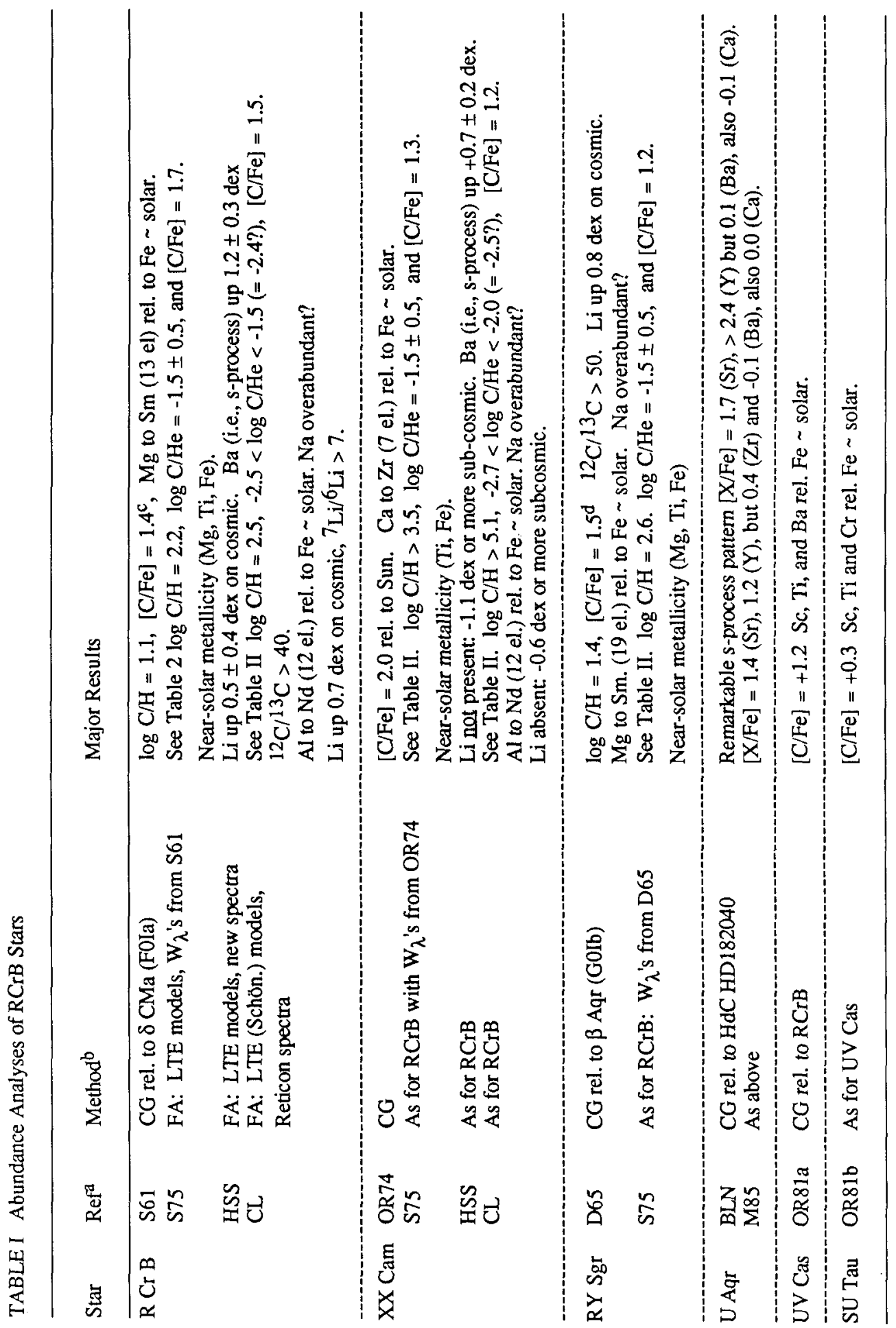


Notes to Table I

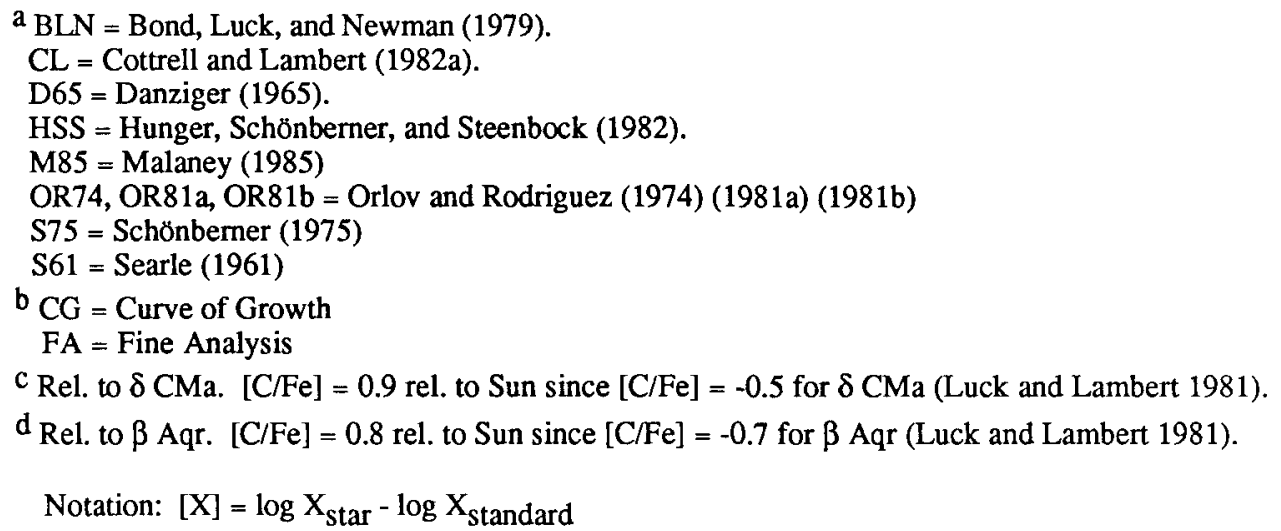

\section{The R Coronae Borealis Stars}

\subsection{Introduction}

In 1985, we celebrate the fiftieth anniversary of the publication of Berman's (1935) pioneering analysis of $\mathrm{RCrB}$. Modern quantitative spectroscopy of $\mathrm{RCrBs}$ began with Searle's (1961) differential curve of growth analysis of $\mathrm{RCrB}$. The principal characteristics of the chemical composition of $\mathrm{RCrBs}$ are summarized in Table I. This table and the ensuing discussion draws upon no more than a dozen papers - the total production over fifty years by a handful of spectroscopists.

Here, I comment on the following abundance ratios and the associated questionsof nucleosynthesis:

- $\mathrm{C} / \mathrm{He}$

$-\mathrm{C} / \mathrm{H}$

$-\mathrm{Li}$

- CNO

- $\mathrm{Na}$ to $\mathrm{Ni}$

- Heavy ( $>\mathrm{Fe})$ elements
How is this to be determined when He may be undetectable in the spectrum?

Is a trace of $\mathrm{H}$ always present?

Is $\mathrm{Li}$ present in $\mathrm{RCrBs}$ ?

How are these key ratios determined?

Is the ratio of odd and even ( $\alpha$ ) light elements to the Fe-group representative of young or old disk stars? Are s-process elements (e.g. Ba) overabundant (rel. to $\mathrm{Fe})$ ?

\subsection{The $\mathrm{C} / \mathrm{He}$ Ratio}

The atmosphere of a RCrB star is severely $\mathrm{H}$ deficient (Bidelman 1953). A plausible assumption is that the atmosphere is now dominated by nuclear processed material in which the initial $\mathrm{H}$ was converted to $\mathrm{He}$. The presence of an extraordinary number of $\mathrm{C} \mathrm{I}$ lines in the spectra would seem to imply that some of the synthesized He may have been burnt to $\mathrm{C}$ and, perhaps, $\mathrm{O}$ and other light $\alpha$-rich elements. Since there is no a priori argument by which to determine the $\mathrm{C} / \mathrm{He}$ ratio, a spectroscopic derivation is necessary.

Beginning students of stellar spectroscopy quickly appreciate that the abundance ratio $\mathrm{X} / \mathrm{H}$ is obtainable from a line of element $\mathrm{X}$ in the spectrum of a normal star without recourse to a measurement of a hydrogen line. Of course, the continuum is that "line" because hydrogen controls the continuous opacity. What is the corresponding opacity in 
RCrBs? Searle's (1961) tentative identification of the photoionization of neutral carbon was confirmed by Schönberner (1975) who constructed model atmospheres. Helium is a minor source of opacity unless the $\mathrm{C} / \mathrm{He}$ is very small. Furthermore, photospheric He may not contribute absorption lines to the spectra of these rather cool stars. In short, He may be undetectable, but yet the most abundant element. With $\mathrm{C}$ as the major source of continuous opacity $\left(\kappa_{c}\right)$, it is the abundance ratio $\mathrm{X} / \mathrm{C}$ that is provided by analysis of lines of element $\mathrm{X}$. Unless photospheric $\mathrm{He} \mathrm{I}$ lines are identifiable, one is restricted to setting upper and lower limits on the $\mathrm{C} / \mathrm{He}$ ratio (S75).

The plausible assumption that the mass fraction of intermediate elements $(Z \geq 16)$ has not been changed by nuclear burning is employed to set an upper limit on the $\mathrm{C} / \mathrm{He}$ ratio. Line analysis gives the ratio $r_{x}(C r B)=f_{x} / f_{c}$, where $f$ denotes a mass fraction and $X$ is an element heavier than oxygen. Since the $\mathrm{RCrB}$ stars do not belong to the extreme Pop. I, we may suppose that their initial metal content did not exceed the solar value. Their Galactic distribution - a marked concentration towards the Galactic center - suggests an old-disk population. Then

or

$$
f_{x}(R C r B)=r_{x}(R C r B) f_{c}(R C r B) \leq f_{x}(\odot)
$$

$$
\mathrm{f}_{\mathrm{c}}(\mathrm{RCrB}) \leq \mathrm{f}_{\mathrm{x}}(\odot) / \mathrm{r}_{\mathrm{x}}(\mathrm{RCrB})
$$

In this way, Schönberner (1975) set a limit $\mathrm{C} / \mathrm{He} \leq 0.10$. Later, Cottrell and Lambert (1982a, here CL), who had access to high S/N Reticon spectra, gave lower limits: $\mathrm{C} / \mathrm{He} \leq 0.03(\mathrm{RCrB})$ and $\leq 0.01(\mathrm{XX} \mathrm{Cam})$ where $\mathrm{f}_{\mathrm{He}}+\mathrm{f}_{\mathrm{C}}+\mathrm{f}_{\mathrm{N}}+\mathrm{f}_{\mathrm{O}} \approx 1$. The difference in part reflects the lower continuous opacity employed by CL. However, the major factor is their use of much weaker lines, which are less dependent upon the microturbulence.

A lower limit to $\mathrm{C} / \mathrm{He}$ is provided by the observed equivalent widths of $\mathrm{C} I$ lines of known oscillator strength. If $\mathrm{C} / \mathrm{He}$ exceeds some minimum value, then $\mathrm{C}$ is the dominant contributor to $\kappa_{c}$ and the equivalent width of a C I or C II line is then effectively independent of $\mathrm{C} / \mathrm{He}$. The equivalent width is, of course, affected by the effective temperature, the surface gravity, and microturbulence. If $\mathrm{C} / \mathrm{He}$ is smaller than a certain value, the contribution of $C$ to $\kappa_{c}$ is negligible and the strength of a $C$ line is dependent on the $\mathrm{C} / \mathrm{He}$ ratio. Observed equivalent widths $\left(\mathrm{W}_{\lambda} \mathrm{s}\right)$ of weak $\mathrm{C}$ lines then demand a minimum value of $\mathrm{C} / \mathrm{He}$. Schönberner set $\mathrm{C} / \mathrm{He} \geq 0.01$ in $\mathrm{RCrB}, \mathrm{XX} \mathrm{Cam}$, and $\mathrm{RY} \mathrm{Sgr}$ from observations of strong $C I$ lines. With access to weak $C I$ lines, CL obtained lower limits: $\mathrm{C} / \mathrm{He} \geq 0.003(\mathrm{RCrB})$ and $0.002(\mathrm{XX} \mathrm{Cam})$. These limits are almost independent of the adopted microturbulent velocity and the atmospheric parameters.

The $\mathrm{C} / \mathrm{He}$ ratio also affects the atmospheric structure. Hunger, Schönberner, and Steenbock (1982, here HSS) note that the residual line intensity in the cores of strong lines is sensitive to the $\mathrm{C} / \mathrm{He}$ ratio through its influence on the atmospheric structure. A fit to the $\mathrm{Li}$ I $6707 \AA$ doublet in $\mathrm{RCrB}$ gave $\log \mathrm{C} / \mathrm{He}=-2.0$ (HSS). Earlier, Schönberner had adopted $\log \mathrm{C} / \mathrm{He}=-1.5 \pm 0.5$. CL's limits were $-2.5 \leq \log \mathrm{C} / \mathrm{He}<-1.5$ for $\mathrm{RCrB}$.

Keenan and Greenstein (1963) reported the presence of the He I line $5876 \AA$ in the spectrum RCrB. CL confirmed this identification and showed that the line is considerably stronger in XX Cam and RY Sgr. If this is a photospheric line and formed in LTE, it gives a direct estimate of the $\mathrm{C} / \mathrm{He}$ ratio. This abundance would seem to be very dependent on the adopted effective temperature, but this dependence is reduced considerably by analyzing the $\mathrm{He}$ I lines relative to the $\mathrm{C} \mathrm{II} \mathrm{lines.} \mathrm{The} \mathrm{resulting} \mathrm{C} / \mathrm{He}$ ratios of $0.004(\mathrm{RCrB})$ and $0.003(\mathrm{XX} \mathrm{Cam})$ given by $\mathrm{CL}$ are close to their above lower limits. Unfortunately, the chromosphere may contribute to the $5876 \AA$ triplet. Querci and 
Querci (1978) reported the He I $10830 \AA$ line to have a P Cygni profile in a spectrum of $\mathrm{RCrB}$ taken 1 mag below maximum light. They attributed the line to the circumstellar shell. In contrast, Zirin (1982) reports the He I $10830 \AA$ line to be absent $\left(\mathrm{W}_{\lambda} \leqslant 70 \mathrm{~m} \AA\right.$ ) in his higher dispersion spectra taken in $1978 \mathrm{July}$ when $\mathrm{RCrB}$ was at maximum light. The fact that Zirin's upper limit for the $\mathrm{W}_{\lambda}(10830 \AA)$ is inconsistent with the Querci and Querci (1978) measurement and CL's $W_{\lambda}(5876 \AA$ ) may indicate a variable chromospheric structure. The chromospheric (i.e., NLTE) contribution should be greatly reduced for the singlet $\mathrm{He} \mathrm{I}$ lines. Unfortunately, the $6678 \mathrm{~A}$ line, which is the most promising candidate for detection, is severely blended. CL's rough estimates of its equivalent width correspond to $\mathrm{C} / \mathrm{He}$ ratios only slightly larger than the above lower limits. Further observations of the He I lines in RCrB stars may uncover a variable chromospheric component of the triplet $\left(D_{3}\right.$ and $\left.10830 \mathrm{~A}\right)$ lines. A NLTE study of photospheric helium should be attempted. These lines may yet provide a direct measure of the $\mathrm{C} / \mathrm{He}$ ratio.

\subsection{The $\mathrm{C} / \mathrm{H}$ Ratio}

This ratio is provided by analysis of the Balmer lines. Figure 1 from CL shows the $\mathrm{H} \alpha$ region in XX Cam, RCrB, RY Sgr, and the normal F8 Ia star $\delta \mathrm{CMa}$. H $\alpha$ is undetectable in XX Cam; the line at $6562.1 \AA$, which may be taken for $\mathrm{H} \alpha$ on lower quality spectra, was identified by HSS82 as due to Ti I. The Ti I line is probably responsible for the apparent blueshift of $\mathrm{H} \alpha$ in $\mathrm{RCrB}$ and $\mathrm{RY}$ Sgr. Results for the $\mathrm{C} / \mathrm{H}$ ratio (see Table 1) are available only for the three brightest RCrBs.

Fig. 1 - Spectra of three $\mathrm{RCrBs}$ near $\mathrm{H} \alpha$. The star $\delta \mathrm{CMa}$ is a normal F8 supergiant. Continuum levels are denoted by the short dashed lines. Note the great strength of the CI line and the weakness of $\mathrm{H} \alpha$ in the RCrBs.

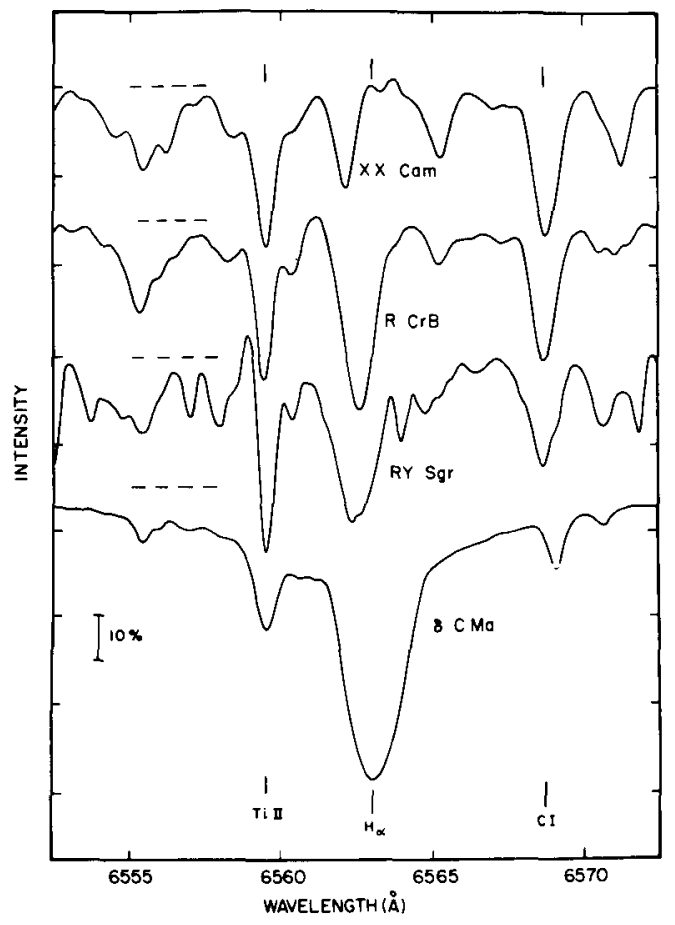


Published $\mathrm{C} / \mathrm{H}$ ratios are entered in Table II where abundances are normalized such that $\sum \mu_{\mathrm{i}} n_{\mathrm{i}}=12.15$ where $\mu_{\mathrm{i}}$ is the mean atomic weight of element $\mathrm{i}$ having a number density $n_{i}$. The numerical value is that for a solar mixture with $H / H e=10$ and $\log n_{H}=$ 12.0. Table II shows that $\mathrm{H}$ in $\mathrm{RCrBs}$ is between 4 to more than 8 orders of magnitude less abundant than in normal stars. It is a trace element with a variable abundance from star to star.

\subsection{Lithium}

Keenan and Greenstein (1963) tentatively identified the Li I 6707 A resonance doublet in $\mathrm{RCrB}$ - an identification confirmed by HSS and CL (Figure 2). Lithium is not present in $\mathrm{XX}$ Cam or RY Sgr. The $6707 \mathrm{~A}$ doublet ion $\mathrm{RCrB}$ is sufficiently strong to permit an estimate of the ${ }^{7} \mathrm{Li} / 6 \mathrm{Li}$ ratio; the wavelength shift between ${ }^{7} \mathrm{Li}$ and ${ }^{6} \mathrm{Li}$ is $0.12 \AA$. CL provided a limit ${ }^{7} \mathrm{Li} / 6 \mathrm{Li}>7$ which suffices to exclude the possibility that the lithium in $\mathrm{RCrB}$ was produced by spallation reactions on the stellar surface, as suggested by HSS following Canal, Isern, and Sanahuja (1977): ${ }^{6} \mathrm{Li} /{ }^{7} \mathrm{Li} \sim 4$ is expected from spallation. The isotopic ratio is consistent with ${ }^{7} \mathrm{Li}$-production by the ${ }^{7} \mathrm{Be}$-transport mechanism: ${ }^{3} \mathrm{He}\left({ }^{4} \mathrm{He}, \gamma\right){ }^{7} \mathrm{Be}\left(\mathrm{e}^{-}, v\right)^{7} \mathrm{Li}$ (Cameron and Fowler 1971). Since there is no comparable mechanism for ${ }^{6} \mathrm{Li}$ production, ${ }^{7} \mathrm{Li} /{ }^{6} \mathrm{Li} \sim \infty$ is predicted.

The observed ratio $\mathrm{Li} / \mathrm{H} \sim 10^{-4}$ for $\mathrm{RCrB}$ excludes the possibility that the $\mathrm{H}$ and $\mathrm{Li}$ are tracers of material which escaped nuclear processing in the star or which was accreted at a late stage. Since the cosmic ratio, $\mathrm{Li} / \mathrm{H} \sim 10^{-9}$, is less than the observed value and normal burning of $\mathrm{H}$ necessarily destroys $\mathrm{Li}$, production of $\mathrm{Li}$ is required to account for a

Fig. 2 - Spectra of three RCrBs and $\delta$ CMa near $6710 \AA$. The Li I $6707 \AA$ is present only in $\mathrm{RCrB}$. The two spectra of RY Sgr show that line doubling at certain phases (the lines are split on JD2444886) may compromise an abundance analysis.

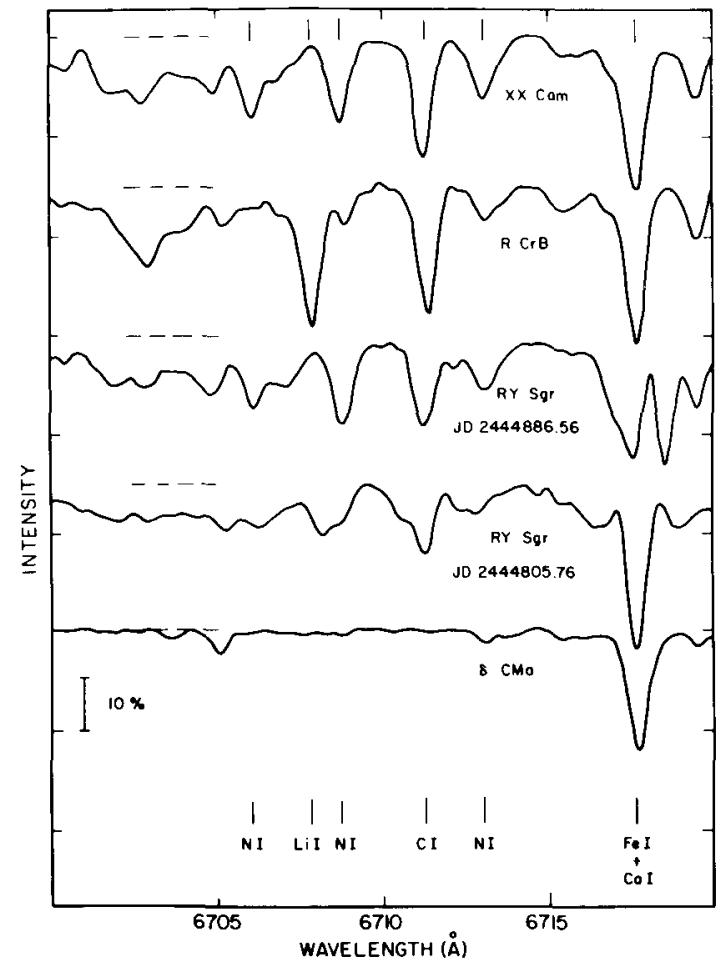


ratio $\mathrm{Li} / \mathrm{H} \sim 10^{-4}$. Since the ${ }^{3} \mathrm{He}$ seed nuclei are produced in fairly copious amounts in the outer envelopes of main sequence stars, the ${ }^{7} \mathrm{Be}$-transport mechanism is a likely source of ${ }^{7} \mathrm{Li}$.

\subsection{Carbon, Nitrogen, and Oxygen}

Since CNO along with $\mathrm{H}$ and $\mathrm{He}$ are expected to be the elements primarily, perhaps exclusively, affected by $\mathrm{H}$ and $\mathrm{He}$ burning, whose products surely dominate the $\mathrm{RCrB}$ envelope, the relative abundances of CNO are clues to the nuclear history of the stars. Both molecular and atomic lines are available for a CNO analysis. To date, a thorough study of the molecular $\left(\mathrm{C}_{2}, \mathrm{CN}\right)$ lines is unavailable; Searle (1961) and Danziger (1965) gave preliminary accounts now needing modernization. Searle obtained neither $\mathrm{N}$ nor $\mathrm{O}$ from atomic lines. Danziger in a differential analysis of RY Sgr relative to $\beta$ Aqr (G0 Ib) gave $[\mathrm{C} / \mathrm{Fe}$ ], [N/Fe], and [O/Fe] from $\mathrm{C} I, \mathrm{~N} I$, and $\mathrm{O} I$ lines. Five $\mathrm{O} I$ lines gave $[\mathrm{O} / \mathrm{Fe}]$ $=-0.6$ with the faint $\mathrm{O} I$ lines in $\beta$ Aqr providing a major uncertainty. For nitrogen, $[\mathrm{N} / \mathrm{Fe}]=0.0$, his analysis is based apparently on one $\mathrm{N} \mathrm{I}$ line referred to the solar spectrum. However, the chosen N I line ( 26484.88$)$ is not present in the solar spectrum. Schönberner's (1975) model atmosphere analysis of RY Sgr includes a discussion of Danziger's C I and O I lines, but not the N I line. These results are given in Table II.

TABLE II Light Elements in RCrB Stars

\begin{tabular}{|c|c|c|c|c|c|c|c|c|}
\hline \multirow[b]{2}{*}{ Star } & \multirow{2}{*}{\multicolumn{2}{|c|}{ Ref. C/He }} & \multicolumn{6}{|c|}{$\log \varepsilon^{a}$} \\
\hline & & & $\mathrm{H}$ & $\mathrm{He}$ & $\mathrm{C}$ & $\mathrm{N}$ & $\mathrm{O}$ & $\mathrm{Fe}$ \\
\hline \multirow[t]{2}{*}{$\mathrm{RCrB}$} & \multirow{2}{*}{$\begin{array}{l}\text { S75 } \\
\text { CL }\end{array}$} & $\begin{array}{c}0.03 \\
0.004\end{array}$ & $\begin{array}{l}7.8 \\
6.6\end{array}$ & $\begin{array}{l}11.5 \\
11.5\end{array}$ & $\begin{array}{r}10.0 \\
9.1\end{array}$ & $\overline{8.1}$ & $\overline{8 .-8}$ & $\begin{array}{l}7.1 \\
6.5\end{array}$ \\
\hline & & 0.03 & 7.5 & 11.5 & 10.0 & 8.9 & 9.7 & 7.4 \\
\hline \multirow[t]{2}{*}{ XX Cam } & \multirow{2}{*}{$\begin{array}{l}\text { S75 } \\
\text { CL }\end{array}$} & $\begin{array}{l}0.03 \\
0.003\end{array}$ & $\begin{array}{l}<6.5 \\
<3.9\end{array}$ & $\begin{array}{l}11.5 \\
11.5\end{array}$ & $\begin{array}{r}10.0 \\
9.0\end{array}$ & $\overline{8.5}$ & $\overline{8.9}$ & $\begin{array}{l}7.5 \\
6.6\end{array}$ \\
\hline & & 0.01 & $<4.4$ & 11.5 & 9.5 & 9.0 & 9.4 & 7.1 \\
\hline RY Sgr & S75 & 0.03 & 7.4 & 11.5 & 10.0 & $8.8^{b}$ & $8.5^{\mathrm{c}}$ & 8.3 \\
\hline \multicolumn{9}{|c|}{$\begin{array}{l}\text { Hot He Stars } \\
\text { BD+10 }\end{array}$} \\
\hline \multirow[t]{2}{*}{ HD124448 } & $8^{\mathrm{H} 83}$ & 0.01 & 8.5 & 11.5 & 9.5 & 8.1 & 8.1 & 6.5 \\
\hline & H83 & 0.01 & $<7.5$ & 11.5 & 9.5 & 8.8 & 8.5 & 7.4 \\
\hline Sun & G84 & 0.005 & 12.0 & 11.0 & 8.7 & 8.0 & 8.9 & 7.7 \\
\hline
\end{tabular}


Notes to Table II

G84 = Grevesse (1984), H83 = Heber (1983). Other references listed at end of Table I

a Normalized to $\Sigma \mu_{\mathrm{i}} \mathrm{n}_{\mathrm{i}}=12.15$

$b$ from Danziger's (1965) estimate that $[\mathrm{C} / \mathrm{Fe}]=+1.5$ and $[\mathrm{N} / \mathrm{Fe}]=0.0$ for $\mathrm{RY}$ Sgr relative to $\beta$ Aqr, Luck and Lambert's (1981) CNO abundances for $\beta$ Aqr, and a normalization to $\log \varepsilon_{\mathrm{c}}=10.0$. This same procedure gives $\log \varepsilon_{\mathrm{o}}=8.3$.

${ }^{c}$ See text for comment on the $O$ abundance.

For $\mathrm{RCrB}$ and XX Cam, CL measured many C I (and a few C II), N I, and O I lines including the low excitation forbidden lines of $\mathrm{C} \mathrm{I}$ and $\mathrm{O}$ I. Abundances obtained using Schönberner's model atmospheres are summarized in Table II. The relative abundances are markedly non-solar:

$\begin{array}{llll} & \text { C } & \text { N } & \text { O } \\ \text { RCrB } & 10 & 1 & 5 \\ \text { XX Cam } & 10 & 3 & 5 \\ \text { Sun } & 10 & 2 & 18\end{array}$

For RY Sgr, Schönberner obtained a lower $\mathrm{O} / \mathrm{C}$ ratio $(\mathrm{O} / \mathrm{C}=0.03)$ from Danziger's equivalent widths. CL's measurements of a selection of C I, N I, and O I lines in RY Sgr indicate that its $\mathrm{O} / \mathrm{C}$ ratio is comparable to that in the other two stars. Additional studies of CNO would be of interest.

The presence of nitrogen suggests that the atmosphere of a $\mathrm{RCrB}$ star contains $\mathrm{N}$ rich material from layers exposed to the $\mathrm{H}$-burning $\mathrm{CNO}$ cycles. The low N/O ratio serves to exclude the possibility that the nitrogen is a tracer of primordial (unprocessed) material. Of course, the dominant fraction of the atmospheric carbon must be a product of He-burning, a conclusion supported by the absence of ${ }^{13} \mathrm{C}$ : ${ }^{12} \mathrm{C} /{ }^{13} \mathrm{C}>40$ for $\mathrm{RCrB}$ (CL), > 50 for RY Sgr (D65). An apparently higher limit may be guessed for U Aqr, the peculiar RCrB star (see below), whose spectrum contains strong ${ }^{12} \mathrm{C}_{2}$ bands and no detectable ${ }^{12} \mathrm{C} /{ }^{13} \mathrm{C}$ bandheads (see $\mathrm{BLN}$ ). A rough limit, ${ }^{12} \mathrm{C} /{ }^{13} \mathrm{C}>100$, may be similarly given for SV Sge (Bidelman 1953).

During the progressive heating of a stellar core prior to He-burning the ${ }^{14} \mathrm{~N}$ synthesized from ${ }^{12} \mathrm{C}$ and ${ }^{16} \mathrm{O}$ by the $\mathrm{CNO}$ cycles in $\mathrm{H}$-burning is converted to ${ }^{18} \mathrm{O}$ whose abundance is then equal to the sum of the initial $\mathrm{CNO}$ abundances. Since some ${ }^{18} \mathrm{O}$ nuclei may survive He-burning and the ensuing mixing, a detection of this isotope could set constraints on models of the birth of RCrBs and related stars. Warner (1967) drew attention to the possible presence of ${ }^{18} \mathrm{O}$ ("nearly all of the $\mathrm{O}$ in the $\mathrm{Hd} \mathrm{C}$ stars is predicted to be in the form of ${ }^{18} \mathrm{O}^{\prime \prime}$ ) and suggested that the infrared vibration-rotation bands of $\mathrm{CO}$ would provide the ${ }^{16} \mathrm{O} / 18 \mathrm{O}$ ratio. To date, these bands have not been detected in $\mathrm{RCrB}$ stars: high resolution spectra at $2.3 \mu \mathrm{m}$ of $\mathrm{RCrB}$ near maximum and near minimum show a continuous spectrum with no evidence of the CO bands $(K$. H. Hinkle and D. L. Lambert, unpublished observations from the KPNO $4 \mathrm{~m}$ FTS). The cooler Hd C star $\mathrm{HD} 182040$ shows $\mathrm{CO}$ in absorption with ${ }^{12} \mathrm{C}^{16} \mathrm{O}$ not ${ }^{12} \mathrm{C}^{18} \mathrm{O}$ as the most abundant species; the ${ }^{16} \mathrm{O} / 18 \mathrm{O}$ ratio is probably rather high. 


\subsection{Sodium to Nickel}

With the persistent exception of sodium, the elements in this group have been shown repeatedly to have solar-like abundance ratios - see Table I. This is not surprising because unevolved (and evolved) disk and halo stars show only minor differences in abundance ratios for this group. For example, abundances of the light $\alpha$-nuclei (e.g. $\mathrm{Mg}, \mathrm{Si}, \mathrm{Ca}$ ) increase to $[\alpha / \mathrm{Fe}] \sim+0.5$ for the halo $([\mathrm{Fe} / \mathrm{H}]<-1)$ with a smooth transition to this value occurring between $-1.0<[\mathrm{Fe} / \mathrm{H}]<-0.3$ (see Tomkin, Lambert, and Balachandran 1985, Nissen, Edvardsson, and Gustafsson 1985; François 1985). CL's analysis of RCrB and XX Cam offers a hint $([\alpha / \mathrm{Fe}] \sim 0.3)$ that the $\alpha$-nuclei have the higher abundance characteristic of the old disk. A thorough reexamination using lines showing minimal or similar sensitivities to the atmospheric structure is recommended because a precise estimate of the $[\alpha / \mathrm{Fe}]$ offers a new probe of the $\mathrm{RCrB}$ stars' initial metallicity.

The odd-mass elements $\mathrm{Na}$ and $\mathrm{Al}$ are predicted to be less abundant in metal-poor stars. For RCrB and XX Cam, the Al abundance (CL) matches that seen in normal dwarfs with $[\mathrm{Fe} / \mathrm{H}]>-1.0$. The $\mathrm{Na}$ overabundance for both stars, $[\mathrm{Na} / \mathrm{Si}] \sim 0.3$, is somewhat at odds with results for the normal dwarfs. Since Na overabundances have been reported for a variety of normal supergiants, we presume that the overabundance reflects a systematic error in the abundance analysis (e.g. the appearance of unaccounted for non-LTE effects).

\subsection{Beyond the Iron Group}

The remarkable star U Aqr apart, the $\mathrm{RCrB}$ stars analysed to date show normal abundances (relative to $\mathrm{Fe}$ ) of the heavy elements; i.e. [s/Fe] $\sim 0.0$ because the selection of elements is dominated by those whose normal origin is ascribed to the s-process. A similar claim for the r-process must be regarded as tentative because it is based apparently on a single line of Eu II measured in RY Sgr D65. A claim by HSS of a Ba overabundance in $\mathrm{RCrB}$ and $\mathrm{XX}$ Cam comparable to that seen in the classical barium stars was disputed by CL who argued that lines chosen by HSS were too strong for a reliable analysis. Weaker lines of Y II and Nd II allowed CL to show that the s-process elements are not overabundant.

Although one can envisage forms of s-processing that produce abundance patterns not yet tested by the abundance analyses, it is clear that the $C$-rich material in the $\mathrm{RCrBs}$ has not come from a cool carbon star in which $[\mathrm{s} / \mathrm{Fe}] \sim 1$ is found for elements from $\mathrm{Sr}$ to the rare-earths. Perhaps one can not yet exclude non-standard s-processing such as the 'mild' s-process suggested by Holweger and Kovács (1984) to account for Sr and Ba enrichment of certain $\mathrm{G}$ and $\mathrm{K}$ supergiants.

The cool high velocity star U Aqr is a RCrB star with a most unusual pattern of sprocess enhancements. BLN discovered and discussed the pattern; the Sr overabundance is so large that the Sr II $4077 \AA$ line rivals the $\mathrm{Ca} \mathrm{II} \mathrm{H}$ and $\mathrm{K}$ lines in strength. Further observations and discussion are given by Malaney (1985). In U Aqr (see Table I), the progression of overabundances in the consecutive elements $\mathrm{Sr}, \mathrm{Y}$ and $\mathrm{Zr}$ is remarkable; $\mathrm{Sr}$ and $\mathrm{Y}$ are greatly overabundant, but $\mathrm{Zr}$ is only slightly enhanced. Furthermore, $\mathrm{Ba}$ is not enhanced.

Such a pattern for the overabundances suggests that exposure of the Fe-group seed nuclei to star neutrons has been slight. If the integrated exposure is assumed to follow an exponential distribution $\exp \left(-\tau / \tau_{\mathrm{o}}\right)$ where

$$
\tau=\int N(n) v d t
$$

and $\tau_{0}$ is an adjustable scale factor and $N(n)$ is the neutron density, Malaney (1985) finds that $\tau_{\mathrm{o}} \sim 0.1 \mathrm{mb}^{-1}$ fits the observed pattern of abundances. This mild exposure converts 
few of the Fe-group 'seed' nuclei to heavier elements so that high $\mathrm{Sr}, \mathrm{Y}$ and normal Fegroup abundances are possible even though the entire atmosphere may have been exposed to neutrons. BLN consider a single exposure rather than an exponential distribution and find that $\tau \sim 0.6 \mathrm{mb}^{-1}$ fits their observed overabundances. At this exposure, the Fe-group are depleted significantly at the s-process site. Since U Aqr is presumed to have a normal Fe abundance, BLN's choice of a single exposure requires that the present atmosphere be a mixture of neutron-processed and unprocessed material.

Identification of the s-processing event with a location on a stellar evolutionary track is tantamount to speculation. BLN suggest that an exceptionally violet helium core flash in a low-mass star stimulated mixing leading to ignition of a neutron source (presumably ${ }^{13} \mathrm{C}(\alpha, n)^{16} \mathrm{O}$ ), and ejection of the H-rich envelope. Malaney prefers to locate the birth of $U$ Aqr on the asymptotic giant branch (AGB) where He-shell flashes are supposed to be responsible for the s-processing. Continued evolution with mass loss via a stellar wind on the AGB or envelope ejection to form a planetary nebula result in a white dwarf having a thin hydrogen-rich envelope. A final He shell flash is predicted to consume the envelope and to restore the star for a short time to a position close to the $\mathrm{AGB}$ with, perhaps, a conposition characteristic of $\mathrm{RCrBs}$ in general and $\mathrm{U}$ Aqr in particular (Renzini 1979, 1981; Iben 1984). The s-processing charachteristic of U Aqr may occur in this final He shell flash.

To constrain these and future speculations, it will be necessary, as noted by BLN and Malaney, to complete a thorough abundance analysis of U Aqr. Both published studies combine just a few very strong lines and a crude differential analysis relative to the Hd C star HD182040. A thorough search for additional heavy elements could yield new information. The $\mathrm{Rb} / \mathrm{Sr}$ ratio could indicate the neutron density at the s-process site (Tomkin and Lambert 1983). While technetium may be present if the s-processing occurred in a He-shell flash, it should have decayed if the core flash was responsible for the birth of a He main sequence star which subsequently evolved to the $\mathrm{RCrB}$ stage. The Fe-group and lighter elements should be investigated for abundance anomalies arising from mild s-processing. Such an extended analysis should be made of the other high velocity and the fainter low velocity stars.

\subsection{Nuclear History and Stellar Evolution}

The history of nuclear processing, mixing, and mass loss is recorded in the chemical composition of the atmosphere. I discuss this composition in terms of processing wrought by the CNO cycles and the $3 \alpha$ process. Next, I comment on the similarities and differences in composition between the RCrB's and the hot He stars. Finally, I sketch possible origins for RCrB's.

Qualitatively, the composition of the RCrB stars (Table II) is that of a He and Nrich zone (in which essentially all the $\mathrm{H}$ has been processed through the $\mathrm{CNO}$ cycle), into which $3 \alpha$ processed material has been mixed. A production scenario for the $\mathrm{RCrB}$ variables must account for the paucity of these objects in the Galaxy; i.e., the evolution through this phase must be extremely rapid and/or the $\mathrm{RCrB}$ structure is produced in only a small percentage of stars.

The remarkable $\mathrm{H}$ deficiency shows that the fraction of pristine material in the atmosphere is very slight. I assume that nuclear-processed material dominates the atmosphere, and that the iron-group nuclei have not been affected by processing. I assume that the abundances relative to iron were initially solar. This initial composition is summarized in Table III with the observed abundances for RCrB and XX Cam. 
A stellar core is first affected by $\mathrm{H}$-burning through the $\mathrm{p}$-p chain and the CNO cycles. Prior to the onset of He burning, the CNO cycles have run to equilibrium even though the $\mathrm{p}$-p chain may have been the dominant energy source. A low mass star will reach $\mathrm{T} \sim 60 \times 10^{6} \mathrm{~K}$ in the core where the equilibrium abundances are ${ }^{12} \mathrm{C} /{ }^{14} \mathrm{~N} \approx 0.03$, ${ }^{16} \mathrm{O} /{ }^{14} \mathrm{~N} \approx 0.005$, and ${ }^{12} \mathrm{C} /{ }^{13} \mathrm{C}=3.4$ (Fowler, Caughlan, and Zimmerman 1975). Li is completely destroyed. The composition of the He-rich core prior to $\mathrm{He}$ ignition is given in Table III under the heading "CNO"; the key point is that it is $\mathrm{N}$ rich and $\mathrm{C}$ and $\mathrm{O}$ poor.

TABLE III Observed and Predicted Compositions (after CL)

\begin{tabular}{|c|c|c|c|c|c|c|}
\hline \multirow[b]{3}{*}{ Element } & \multicolumn{6}{|c|}{ Abundance Ratio $\log (\mathrm{X} / \mathrm{Fe})$} \\
\hline & \multirow[b]{2}{*}{ Sun } & \multicolumn{3}{|c|}{ Stellar Core } & \multirow[b]{2}{*}{$\mathrm{RCrB}$} & \multirow[b]{2}{*}{ XX Cam } \\
\hline & & $\overline{\mathrm{CNO}}$ & $\mathrm{He}^{\prime}$ & $\mathrm{He}^{\prime \prime}$ & & \\
\hline $\mathbf{H}$ & 4.5 & $\mathrm{XXX}^{\mathrm{b}}$ & XXX & XXX & 0.2 & $<-2.6$ \\
\hline $\mathrm{He}^{\mathrm{c}}$ & 3.3 & 4.0 & 3.9 & 5.0 & $5.1(4.2-5.2)$ & $4.8(4.0-5.0)$ \\
\hline${ }^{12} \mathrm{C}$ & 1.2 & 0.1 & 2.6 & 2.6 & 2.7 & 2.4 \\
\hline${ }^{13} \mathrm{C}$ & -0.7 & -0.4 & -0.5 & -1.5 & $<1.1$ & $\ldots$ \\
\hline $14 \mathrm{~N}$ & 0.5 & 1.6 & 1.5 & 1.9 & 1.6 & 1.9 \\
\hline $16 \mathrm{O}$ & 1.4 & -0.7 & 2.2 & 2.2 & 2.3 & 2.2 \\
\hline
\end{tabular}

a The predicted composition is shown for the following exposures to nuclear burning: $\mathrm{CNO}=$ post $\mathrm{H}$-exhausted core collapse with $\mathrm{CNO}$ cycles run to equilibrium for $\mathrm{T} \sim 60 \mathrm{x}$ $10^{6} \mathrm{~K} . \mathrm{He}^{\prime}=$ post $\mathrm{He}$-core flash with the 3 a process converting $20 \%$ of the $\mathrm{He}$ to $\mathrm{C}$ and $\mathrm{O}$ in the proportions 2 to 1 . $\mathrm{He}^{\prime \prime}=$ as for He' but the $\mathrm{H}$-exhausted core was originally metal poor (i.e., an old disk star) by a factor of 10 with a modest $O$ overabundance (Clegg, Lambert, and Tomkin 1981). $2 \%$ of the $\mathrm{He}$ is burnt to $\mathrm{C}$ and $\mathrm{O}$.

b XXX denotes a very small abundance ratio X/Fe.

$\mathrm{c}^{\mathrm{F}}$ For $\mathrm{RCrB}$ and $\mathrm{XX}$ Cam, upper and lower limits (see $\$ 2.2$ ) are shown in parentheses.

The onset of $\mathrm{He}$ burning converts $\mathrm{He}$ to ${ }^{12} \mathrm{C}$, destroys ${ }^{14} \mathrm{~N}$ by the ${ }^{14} \mathrm{~N}(\alpha, \gamma){ }^{18} \mathrm{~F}\left(\mathrm{e}^{+}, \nu\right)^{18} \mathrm{O}$ reactions, and may produce ${ }^{16} \mathrm{O}$. When ${ }^{16} \mathrm{O}$ production occurs, the ${ }^{18} \mathrm{O}$ will be destroyed by ${ }^{18} \mathrm{O}(\alpha, \gamma)^{22} \mathrm{Ne}$ and ${ }^{18} \mathrm{O}(\alpha, \mathrm{n})^{21} \mathrm{Ne}$ reactions. Since the burning may be inhomogeneous through the core, some ${ }^{18} \mathrm{O}$ may survive and be present in $\mathrm{H}$-poor, $\mathrm{C}$-rich atmospheres. The ${ }^{16} \mathrm{O}$ production depends on the core temperature and the duration of He burning. The rate for the crucial ${ }^{12} \mathrm{C}(\alpha, \gamma)^{16} \mathrm{O}$ reaction is uncertain. Since ${ }^{14} \mathrm{~N}$ is readily destroyed in He-burning, the observed high $\mathrm{N}$ abundance demands that the atmosphere now contain a mixture of CNO-cycle exposed and $3 \alpha$-processed material. Table III shows predictions for two cases of partial He burning with the arbitrary stipulation that the $\mathrm{C}$ and $\mathrm{O}$ be produced in the proportions 2 to 1 . The (uncertain) $\mathrm{C} / \mathrm{He}$ ratio derived from the $5876 \AA$ line would seem to imply that the intial star was moderately metal deficient. The quantitative correspondence between the $\mathrm{He}^{\prime}$ and He" predictions in Table III and the observed abundance is not as significant as the general constraints set by the abundances; the atmospheres contain a large amount of $\mathrm{He}$ 
previously exposed to the $\mathrm{CNO}$ cycles with a small mixture of the products of $\mathrm{He}$ burning under conditions adequate to produce ${ }^{16} \mathrm{O}$. Li preduction is attributed to the ${ }^{7} \mathrm{Be}$-transport mechanism (see § 2.4).

A nuclear history must also explain why s-processing has not occurred in $\mathrm{RCrB}$ and XX Cam. The s-processing in a He core or shell is most probably controlled by the neutron sources ${ }^{22} \mathrm{Ne}(\alpha, n){ }^{25} \mathrm{Mg}$ and ${ }^{13} \mathrm{C}(\alpha, n){ }^{14} \mathrm{O}$. The initial ${ }^{22} \mathrm{Ne}$ abundance is low, and an insufficient neutron flux ( $<1$ per Fe seed nucleus) is supplied. Additional ${ }^{22} \mathrm{Ne}$ nuclei may be created from ${ }^{14} \mathrm{~N}$ via ${ }^{18} \mathrm{O}$ and ${ }^{18} \mathrm{O}(\alpha, \gamma) 22 \mathrm{Ne}$. Then, the maximum supply is 30 to 60 neutrons per Fe seed nucleus; the higher figure pertains to metal-poor cores. It is interesting to note that BLN report that an exposure of 30 neutrons per Fe seed nucleus is required to explain the s-process enhancements in U Aqr. Consumption of ${ }^{22} \mathrm{Ne}$ is preventable by limiting the core temperature; if $\mathrm{T} \leq 2 \times 10^{8} \mathrm{~K},{ }^{16} \mathrm{O}$ production seems possible without significant neutron release from ${ }^{22} \mathrm{Ne}$ [note that at low temperatures ${ }^{22} \mathrm{Ne}(\alpha, \gamma)^{26} \mathrm{Mg}$ is favored over ${ }^{22} \mathrm{Ne}(\alpha, \mathrm{n}){ }^{25} \mathrm{Mg}$.

The residual abundance of ${ }^{13} \mathrm{C}$ in the He burning core or shell is too low to cause significant s-processing by neutrons released through the ${ }^{13} \mathrm{C}(\alpha, n)^{16} \mathrm{O}$ reaction at $\mathrm{He}$ burning. The neutron supply may be enhanced by mixing in a little $\mathrm{H}$-rich material so that ${ }^{13} \mathrm{C}$ is regenerated and neutrons created from fresh ${ }^{12} \mathrm{C}$ by the chain ${ }^{12} \mathrm{C}\left(\mathrm{p}, \mathrm{e}^{+}\right)^{13} \mathrm{C}(\alpha, n)^{16} \mathrm{O}$. As long as the number of protons added is small, such mixing will not produce an inadmissible reduction of the ${ }^{12} \mathrm{C} /{ }^{13} \mathrm{C}$ ratio. This latter proviso is physically plausible because the large energy release, which must accompany the addition of protons, may be presumed to quench the mixing. For $\mathrm{RCrB}$ and $\mathrm{XX}$ Cam, I suggest that protons were not mixed into the He-burning region and, hence, s-processing did not occur.

This sketch of the nuclear history of the $\mathrm{RCrB}$ atmosphere must be integrated into a full evolutionary history for these unusual stars, which probably consist of a low mass $\left(\mathrm{M} \sim 1 \mathrm{M}_{\odot}\right)$ star with a thin He-rich envelope around a carbon-oxygen core. One may reasonably suppose that the $\mathrm{RCrB}$ are related to the hot extreme He stars. Schönberner (1975) notes that evolutionary tracks for pure He stars evolving to the red giant region follow similar paths in the H-R diagram on their ascending and descending branches. With present uncertainties in the stars effective temperatures and surface gravities, one cannot rely on location in the H-R diagram to determine whether the hot He stars are the progenitors or descendants of RCrBs. Heber and Schönberner (1981) claimed that the observed galactic density of $\mathrm{RCrB}$ and $\mathrm{He} \mathrm{B}$ stars is consistent with evolution from supergiant (RCrB) to dwarf (He B star) as modeled by Schönberner (1977). The changing pulsational period of RY Sgr confirms this suggestion (Kilkenny 1982).

Whatever the precise relation between the $\mathrm{RCrB}$ and $\mathrm{He} \mathrm{B}$ stars, one might expect them to have similar surface compositions. However, one should recognize that very few stars ( $3 \mathrm{RCrBs}, 4 \mathrm{He} \mathrm{Bs}$ ) have been analysed in detail; we may not yet know the composition of the typical $\mathrm{RCrB}$ or He B star. In Table II, I include data from Heber (1983) for two He Bs: HD124448 and the metal-poor BD $+10^{\circ} 2179$. For the He B stars, the $\mathrm{C} / \mathrm{He}$ ratio is directly obtainable from absorption lines. Heber's (1983) four stars have $\log \mathrm{C} / \mathrm{He}=-2.0$ to -2.2 , a value slightly higher than CL's $\mathrm{D}_{3}$-based results for $\mathrm{RCrB}(-2.4)$ and XX Cam (-2.5) and less than the compromise $(-1.5)$ adopted in S75. The presence of a metal-poor star (initial $[\mathrm{Fe} / \mathrm{H}] \sim-1$ ) in the sample of four He B stars certainly encourages the view that some $\mathrm{RCrBs}$ may also be metal-poor. On the assumption that their $\mathrm{C} / \mathrm{He}$ ratio is close to CL's derived lower limit, we see that the compositions of $\mathrm{RCrB}, \mathrm{XX} \mathrm{Cam}$, and $\mathrm{BD}+10^{\circ} 2179$ are similar except for $\mathrm{H}$ and $\mathrm{O}$. 
Hydrogen is evidently variable from star-to-star in both classes. Oxygen is apparently rather more abundant in $\mathrm{RCrBs}$ than in $\mathrm{BD}+10^{\circ} 2179$; the earlier and lower estimate for $\mathrm{RY} \mathrm{Sgr} \mathrm{(S75)} \mathrm{is} \mathrm{in} \mathrm{good} \mathrm{agreement} \mathrm{with} \mathrm{that} \mathrm{for} \mathrm{BD}+10^{\circ} 2179$. If the $\mathrm{RCrBs}$ are assumed to have solar metal abundance, their composition matches that of $\mathrm{He} B$ stars (HD168476, 124448, and BD $-9^{\circ} 4395$ ) except for oxygen which is about an order of magnitude higher in RCrBs according to CL (but not S75). Clearly, oxygen deserves further study.

What is the origin of RCrB stars? The AGB is an attractive location for the first appearance of He-rich stars. Envelope instabilities (He shell flashes) affecting AGB stars are possibly responsible for mass ejection leading to the formation of planetary nebulae. Härm and Schwarzschild (1975) shows that such instabilities could leave the star with a very thin $\mathrm{H}$ envelope. Continued mass loss either as a steady wind or in bursts would produce a He-rich star. In addition, $\mathrm{H}$ may be consumed between shell flashes (Paczyński 1971). Thus one could envisage a connection between the RCrB stars and the He and C-rich ejecta of two planetary nebulae, Abell 30 and 78 (Jacoby and Ford 1983).

Ejection of the H-rich envelope through explosion may also occur at the He core flash, as a low-mass $\left(<2.5 \mathrm{M}_{\odot}\right)$ star reaches the tip of the first red giant branch. Deupree and Cole (1981) in a hydrodynamical analysis of the He-core flash show that, although a prediction of the nucleosynthesis is beset with considerable uncertainties, a substantial amount of ${ }^{4} \mathrm{He}$ is processed to ${ }^{12} \mathrm{C}$, and a fraction is further processed to ${ }^{16} \mathrm{O}$. They suggest that significant mass loss could occur at the He core flash. The stellar remnant is probably a He star which evolves to the RCrB stage.

The above scenarios presume that RCrBs and He B stars evolve from single stars. Recently, Iben and Tutukov, (1985, here IT) proposed that these He-rich stars are created by the merger of a pair of white dwarfs with the less massive star having a He core with a thin surface layer of $\mathrm{H}$, and the more massive white dwarf having a $\mathrm{C}-\mathrm{O}$ core. IT argue that the merger results in a star with a surface composition like that of a $\mathrm{RCrB}$ : the $\mathrm{He}$ white dwarf forms a coating around the $\mathrm{C}-\mathrm{O}$ dwarf. The $\mathrm{N}$ comes from the He white dwarf where the $\mathrm{N}$ was synthesized during $\mathrm{H}$ burning from the initial $\mathrm{CNO}$. The surface $\mathrm{C}$ and $\mathrm{O}$ of the $\mathrm{RCrB}$ star is presumed to be dredged up during the merger from the underlying $\mathrm{C}-\mathrm{O}$ dwarf. Abundance variations from star to star are attributable to variations in stellar masses and in the dredge-up of $\mathrm{C}$ and $\mathrm{O}$ which, in turn, is presumed to be a function of the stellar masses. The immediate product of the merger is expected to be a RCrB star which later evolves to a He B star, as suggested by Heber and Schönberner's (1981) study. Although a massive and short-lived star is needed to create the C-O white dwarf, the much longer time scale for production of a $\mathrm{RCrB}$ is set by the orbital decay induced by emission of gravitational waves. IT note that their scenario can account for the observed number of giant He stars. This attractive feature of the IT model sets it apart from the speculations assigning the birth of Hd's to either the AGB or the tip of the Hecore flash at the tip of the red giant branch. In such alternative speculations, special and as yet unidentified selection effects must be invoked to ensure that Hd stars are created in the observed numbers as the vast majority of stars evolve through the He-core flash and along the AGB without major changes in surface $H$ content.

\subsection{Concluding Comments}

My present emphasis on chemical composition should not be misinterpreted as an indication that the photospheric structure of a $\mathrm{RCrB}$ star is now devoid of interest for spectroscopists. Their abrupt and unpredictable declines to minimum light remain a puzzle. The key to the puzzle may lie in spectroscopic studies of the photospheric 
pulsations (Cottrell and Lawson, this conference) which appear as line shifts, asymmetries and even line doubling over narrow phase intervals (Cottrell and Lambert 1982b).

With the advent of large telescopes equipped with efficient spectrometers and a resurgence of theoretical work on the creation and evolution of peculiar stars, abundance analyses are certain to be extended from the brighter RCrBs to the majority of the 40 or so galactic members of the class and to the brighter examples now identified in the Magellanic Clouds. These analyses should be comprehensive in order that the nuclear history can be unravelled; in particular, $\mathrm{He}, \mathrm{Li}, \mathrm{C}, \mathrm{N}$, ) and the s-process elements should be included. Similarly thorough studies should be made of the Hd C and He B stars.

\section{The Hydrogen-Deficient Carbon (Hd C) Stars}

In 1953, Bidelman (1953) coupled the RCrB stars with four non-variable cooler stars to form a class of "apparently hydrogen-deficient stars which appear also to be rich in carbon". I refer to the non-variables as Hd C stars. The prototype may be taken to be HD182040 whose spectral peculiarities were suspected first by Rufus (1915). Today, five stars comprise the class (HD137613, 148839, 173409, 175893, and 182040); HD148839 was identified as a Hd C star by Warner (1967). XX Cam ("The Inactive RCrB Star" - Rao, Ashok, and Kulkarni 1980), which has shown just one minimum in 90

TABLE IV Composition of Hd Cs (after Warner, 1967)

\begin{tabular}{|c|c|c|}
\hline Quantity & Result & Comments \\
\hline$\frac{\mathrm{C}}{\mathrm{He}}$ & $\sim 10^{-2}$ & $\begin{array}{l}\text { Uncertain - see comments on this ratio for } \\
\text { RCrBs. }\end{array}$ \\
\hline$\frac{\mathrm{C}}{\mathrm{H}}$ & $210^{3}$ & $\begin{array}{l}\text { For } \mathrm{HD} 148839, \text { which Warner proposed as } \\
\text { intermediate between normal cool carbon } \\
\text { stars and the } \mathrm{Hd} \mathrm{Cs} \mathrm{C} / \mathrm{H} \sim 0.05 \text {. }\end{array}$ \\
\hline$\left[\frac{\mathrm{C}}{\mathrm{Fe}}\right]$ & $0.8 \mathrm{dex}$ & $\begin{array}{l}\text { The four extreme } \mathrm{Hd} \mathrm{Cs} \text { have }[\mathrm{C} / \mathrm{Fe}] \approx 0.7 \\
\text { to } 1.0 \text { dex. HD148839 has }[\mathrm{C} / \mathrm{Fe}]=0.5 \\
\text { dex. } \mathrm{RCrBs}(\mathrm{CL}) \text { have }[\mathrm{C} / \mathrm{Fe}] \sim 1.2 \text { to } 1.5 \text {. }\end{array}$ \\
\hline $\mathbf{Z}$ & $(2$ to 0.3$) \odot$ & $\begin{array}{l}\text { "Most indications are that the Hd C stars are } \\
\text { slightly metal deficient, in agreement with } \\
\text { their large space velocities." }\end{array}$ \\
\hline $\mathrm{X} / \mathrm{Fe}$ & solar & $\begin{array}{l}\text { With the possible exception of } \mathrm{Na} \text { (see also } \\
\S 2.6), 16 \text { elements }(\mathrm{X}) \text { from Ca to Sm have } \\
\text { [X/Fe] = } 0 \text { to within errors of measurements; } \\
\text { i.e., no s-process enhancement. The lack of } \\
\text { an enhancement for HD148839 suggests that } \\
\text { it is not a close relative of normal carbon } \\
\text { stars for which }[\mathrm{s} / \mathrm{Fe}] \sim 1 \text {. }\end{array}$ \\
\hline${ }^{12} \mathrm{C} /{ }^{13} \mathrm{C}$ & $\geq \quad 100$ & $\begin{array}{l}\text { See Climenhaga (1960), Lambert } \\
\text { (unpublished). }\end{array}$ \\
\hline
\end{tabular}


years, and that just 1.7 mag., deep may be a hybrid, part $\mathrm{RCrB}$ and part $\mathrm{Hd} \mathrm{C}$. If the photospheric pulsations trigger the deep minima characteristic of $\mathrm{RCrBs}$, their relatives lying just outside the instability strip will show very similar spectral characteristics, but lack the variability associated with RCrBs. Although the data on chemical composition are incomplete, the evidence supports Bidelman's bold coupling of $\mathrm{RCrBs}$ and $\mathrm{Hd} \mathrm{Cs}$.

The standard (and only) abundance analysis of Hd Cs is by Warner (1967) who gave a curve of growth analysis of the five members of the class. Equivalent widths of about 500 lines per star spanning the intervals $4225-4570 \AA$ and $4737-4960 \AA$ (the gap is dominated by $\mathrm{C}_{2}$ lines) were measured in each star off $15.6 \mathrm{~A} / \mathrm{mm}$ IIa O plates. Table IV summarizes the principal results which show clearly the similarities between $\mathrm{RCrB}$ and $\mathrm{Hd} \mathrm{C}$ stars. The relative abundances of $\mathrm{CNO}$ are unknown. Warner's prediction that ${ }^{18} \mathrm{O}$ is the dominant oxygen isotope is not confirmed - see $\S 2.5$.

I look forward to new analyses of the Hd Cs using broad spectral coverage and model atmospheres.

\section{Are All Carbon Stars Helium Rich?}

The question posed in the section's title was asked first by Vardya (1966) in a letter to The Observatory. Struck by the fact that hot He stars and $\mathrm{RCrB}$ are C-rich (i.e. C.O $>1$ ) and He-rich, Vardya wondered about other carbon stars. An assertion - "It is very likely that they are also hydrogen deficient and helium enriched" - was followed by an inconclusive discussion of CH-stars. (I shall not discuss $\mathrm{CH}$ stars and the related Barium stars - see Lambert [1985]). I shall comment on the cool carbon stars and the evidence presented recently for a substantial $\mathrm{H}$ deficiency in these AGB stars. To the observer, $\mathrm{H}$ deficiency and He-enrichment seems a plausible malady of an AGB star and, especially, a carbonenriched star which is presumably contaminated with carbon and helium from the Heburning shell. Severe mass loss on the AGB will reduce the thickness of the H-rich envelope whose base may be sufficiently hot to burn hydrogen. The pragmatist would not be surprised to find a range of $\mathrm{H}$ deficiencies on the $\mathrm{AGB}$ whose members may include the immediate progenitors of the RCrB and Hd C stars. In short, the opening question is worthy of examination.

Recent claims that the cool carbon stars are $\mathrm{H}$ deficient are based on model atmosphere based interpretations of observations of $\mathrm{H}_{2}$ quadrupole vibration-rotation lines and the $\mathrm{H}^{-}$flux peak near $1.6 \mu \mathrm{m}$. Goorvitch, Goebel, and Augason (1980) pointed out that the 1-0 S(1) $\mathrm{H}_{2}$ line was not detectable in high resolution spectra of the carbon stars UU Aur and S Cep. Johnson et al. (1983) expanded the search for the $\mathrm{H}_{2}$ line, detected it in C-rich Mira variables (i.e., the coolest stars in their sample), but reported it absent from several warmer carbon stars. In our analysis (Lambert et al. 1986) of infrared spectra of 30 galactic carbon stars, we detected the weaker 1-0 S(0) $\mathrm{H}_{2}$ line in almost all of the sample - see Figure 3. The $S(0)$ line unlike the $S(1)$ line is essentially unblended. Our measurements of the $S(0)$ line are consistent with the $S(1)$ detections reported by Johnson et al. (1983). Here, comparisons with predicted equivalent widths are for the $S(0)$ line.

Across the temperature range spanned by these carbon stars, the dominant form of hydrogen changes from atomic to molecular. The predicted $\mathrm{H}_{2}$ column density as sampled by $\mathrm{H}_{2}$ quadrupole line may be reduced by either introduction of a $\mathrm{H}$ deficiency or a warming of the atmosphere such as might occur through additional opacity in the upper atmosphere. (Other possibilities for reducing the $\mathrm{H}_{2}$ density include departures from LTE and a deep chromosphere.) In the coolest stars, the temperatures favor $\mathrm{H}_{2}$ so strongly that severe warming of an extensive atmosphere is demanded in order to reduce 
the molecule's column density. Hence, one should not be surprised that the $\mathrm{H}_{2}$ lines are seen in the Mira variables with about the predicted strength. All of Johnson et al.'s predictions of $\mathrm{H}_{2}$ lines are based on model atmospheres computed from standard assumptions and including diatomic molecules (principally $\mathrm{C}_{2}, \mathrm{CN}$, and $\mathrm{CO}$ ) as contributors to the line blanketing. Polyatomic moleclues $\left(\mathrm{HCN}, \mathrm{C}_{2} \mathrm{H}_{2}\right)$ resident in the upper atmosphere also contribute to the blanketing (Eriksson et al. 1984) and, hence, to the backwarming of the photosphere. Models constructed using the diatomic and polyatomic contributions to the line opacity provide significantly smaller $\mathrm{H}_{2}$ equivalent widths for the carbon stars. This is illustrated in Figure 3. Our predictions for models lacking $\mathrm{HCN}$ and $\mathrm{C}_{2} \mathrm{H}_{2}$ agree well with those provided by Johnson $e$ t al. from similar but independently computed models. With the inclusion of the polyatomics, the predicted and observed $\mathrm{H}_{2}$ lines are of similar strength; the $\mathrm{H}_{2}$ is seen to be an indicator of the $\mathrm{C} / \mathrm{O}$ ratio, a controlling influence on the abundance of the polyatomics, and the inferred $\mathrm{C} / \mathrm{O}$ ratio is in fair agreement with the ratio determined directly from $\mathrm{C}_{2}$ and $\mathrm{CO}$ lines. Note how the influence of the polyatomics is reduced in the cooler stars where association of $\mathrm{H}$ into $\mathrm{H}_{2}$ is more complete. These calculations, as summarized in Figure 3, show quite clearly that the $\mathrm{H}_{2}$ line is a sensitive probe of the atmospheric structure of these carbon stars. Our improved model atmospheres show it is not necessary to invoke $\mathrm{H}$ deficiency in order to account for the observed strengths of the $\mathrm{H}_{2}$ lines. (Tsuji [1983] has noted that the predicted $\mathrm{H}_{2}$ strengths exceed the observed strengths for late $\mathrm{M}$ giants. We suspect that a

Fig. 3. - Observed and predicted intensity $(\log$ $\left.\mathrm{W}_{\sigma} / \sigma\right)$ of the $\mathrm{H}_{2} 1-0 \mathrm{~S}(0)$ line in cool carbon stars. Observations are represented by filled $\left(\mathrm{T}_{\text {eff }}\right.$ from Tsuji, 1981 , and the infrared flux method) and open ( $\mathrm{T}_{\text {eff }}$ by interpolation) circles. The four stars identified by name are ${ }^{13} \mathrm{C}$-rich. Predictions for models constructed without line blanketing by $\mathrm{HCN}$ and $\mathrm{C}_{2} \mathrm{H}_{2}$ for $\log \mathrm{C} / \mathrm{O}=0.01$ $(\mathrm{C} / \mathrm{O}=1.02)$ and $\log \mathrm{C} / \mathrm{O}=$ $0.13(\mathrm{C} / \mathrm{O}=1.35)$ are given by the broken lines and identified. All other predictions are from models with $\mathrm{HCN}$ and $\mathrm{C}_{2} \mathrm{H}_{2}$ blanketing and for $\log \mathrm{g}=$ $-2,-1$, or 0 and with the $\log$ $\mathrm{C} / \mathrm{O}$ ratios indicated on the right-hand side. Carbon stars have $-1 \leq \log \mathrm{g} \geq 0$.

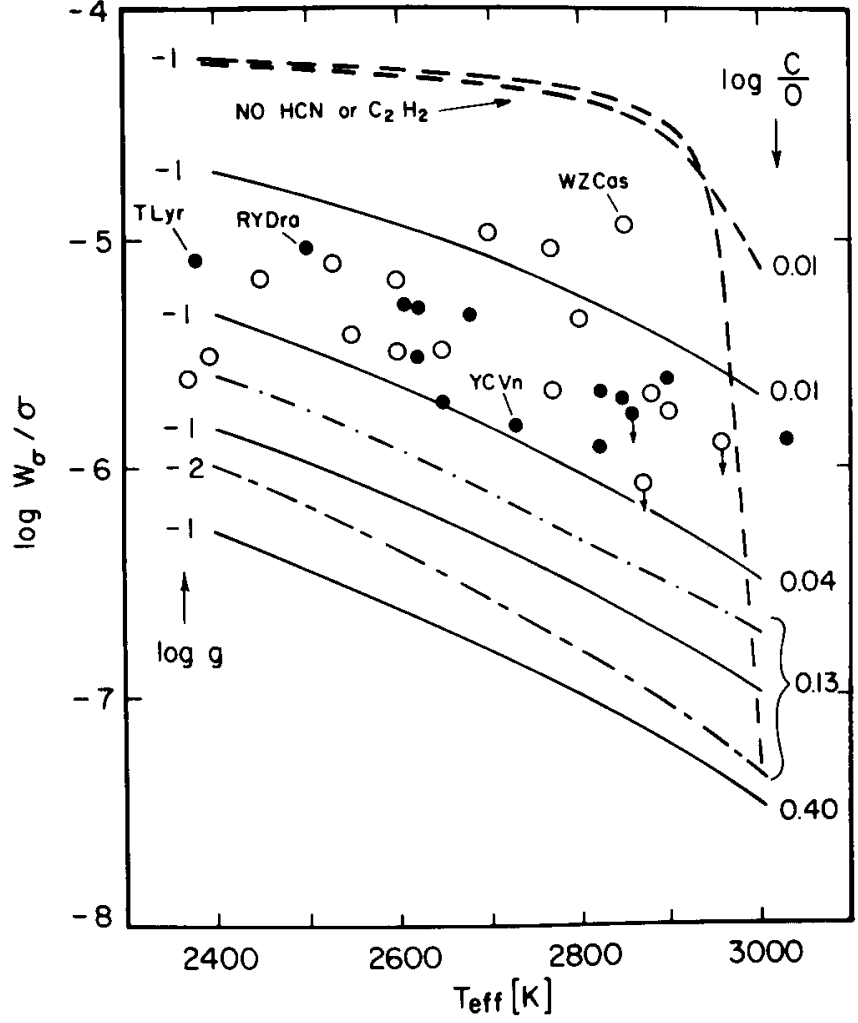


more complete accounting for the line blanketing in the construction of the model atmospheres may remove this discrepancy, too.)

The variation with wavelength of the emergent flux from a star betrays the identities of the principal contributors to the continuous opacity. For a cool star of normal composition, the $\mathrm{H}^{-}$ion dominates the onacity and, hence, the continuum brightness temperature peaks near the $1.6 \mu \mathrm{m}$ opacity minimum. Goebel and Johnson (1984) and Johnson et al. (1985) report that the temperature/flux peak is absent for cool carbon stars and suggest that a $\mathrm{H}$ deficiency of a factor of approximately 10 is required to reconcile predicted and observed fluxes. This deduction is based on predictions for model atmospheres to which diatomic, but not polyatomic, molecules provide line blanketing. Lambert et al. (1986) show that the H-flux peak is disguised by overlying molecular bands and an altered atmospheric structure when lines from polyatomic molecules are included in the blanketing: observed flux curves may be fit by predictions for atmospheres having a normal $\mathrm{H}$ abundance.

In a cool atmosphere, hydrogen deficiency and helium enrichment lower the continuous opacity such that the various atomic and molecular lines from elements other than $\mathrm{H}$ are strengthened even when the elemental mass fractions are unchanged - see Böhm-Vitense (1979) for an elegant discussion. Conversely, if the spectrum of a H-poor star is interpreted on the assumption that the star has a normal $\mathrm{H}$ abundance, the abundance - here $\mathrm{X} / \mathrm{H}$ - will be overestimated. Lambert et al. (1986) derived the metal abundance of their cool carbon stars from several atomic ( $\mathrm{Ca}$ I, FeI) lines: the individual and mean abundances $([\mathrm{Fe} / \mathrm{H}] \sim-0.1$ dex) derived on the assumption that $\mathrm{H}$ is not deficient are consistent with that expected from the kinematics of the stars and with the metal abundance derived from comparable samples of $\mathrm{G}, \mathrm{K}$, and $\mathrm{M}$ giants among whom must be found the progenitors of the cool carbon stars. Dominy, Wallerstein, and Suntzeff (1986) eliminate with thsi same argument the hypothesis that SC stars are H deficient.

If the AGB stars were $H$ deficient, their descendants should suffer the same malady. I identify the planetary nebulae (PN) as the descendants; the ionized shell is part of the AGB star's outer envelope. Some PNs are carbon-rich and I suppose that these are the descendants of the carbon stars; the claim that $\mathrm{C} / \mathrm{O}>1$ for such $\mathrm{PNs}$ rests on analyses of the emission line spectrum and/or the detection of the infrared signature of circumstellar graphite grains. Comparisons between the composition of carbon stars and PNs are restricted by the lack of common elements unaffected by nucleosynthesis and dredge-up on the AGB. Oxygen is one possible example. Lambert et al. (1986) show that the mean $\mathrm{O} / \mathrm{H}$ ratio and its dispersion is the same for M giants (Smith and Lambert 1985, 1986), carbon stars, and the C-rich PNs (Aller and Czyzak 1983). The stellar analyses provide $\mathrm{O} / \mathrm{H}$ from observations of $\mathrm{CO}$ lines and an assumption about the $\mathrm{H}$ abundance. Analyses of $\mathrm{PN}$ provide the $\mathrm{O}$ and the $\mathrm{H}$ abundances directly without an assumption about the $\mathrm{He} / \mathrm{H}$ ratio. Indeed, this latter ratio is measured and shows directly that the PNs are not markedly $\mathrm{H}$ poor: $\mathrm{He} / \mathrm{H}=0.11$ as the mean for $\mathrm{C}$ (and $\mathrm{O}$ ) rich PNs (Aller and Czyzak 1983). Few PNs are significantly enriched in $\mathrm{He}$. A ratio $\mathrm{He} / \mathrm{H}=0.10 \pm 0.01$ is exhibited by local H II regions and young stars (Dufour, Shields, and Talbot 1982; Nissen 1983). Both the $\mathrm{O} / \mathrm{H}$ and $\mathrm{He} / \mathrm{H}$ ratios reinforce our direct arguments that hydrogen is not significantly depleted in a cool carbon star's atmosphere. An enthusiast favoring $\mathrm{H}$-deficient carbon stars is forced to assert that these stars do not evolve into PNs.

In our view, the direct analyses of carbon stars and the indirect appeals to progenitors (G, K, M giants) and descendants (PNs) provide no evidence for a significant ( $>$ a factor of two) $\mathrm{H}$ deficiency in the atmosphers of the carbon stars. Of course, the fact 
that they are not $\mathrm{H}$ deficient and $\mathrm{He}$ enriched translates to a requirement on the $\mathrm{C} / \mathrm{He}$ ratio in the material and the mass of the material added to the envelope from the He burning shell. It appears that such requirements are met by presently available theoretical calculations of the dredge-up (see Lambert et al. 1986). Our answer to Vardya's (1966) question is 'NO'.

\section{An Observer's Ragbag}

This concluding section was stimulated by an inference aired by Luck (1981) in reviewing the implications of his abundance analysis of $R$ Sct, a $R V$ Tauri variable. A standard analysis using a model atmosphere gave $\mathrm{T}_{\mathrm{eff}}=4000 \mathrm{~K}, \log \mathrm{g}=0.0$, and $[\mathrm{Fe} / \mathrm{H}]$ $=-0.9$ and $[\mathrm{X} / \mathrm{Fe}] \sim 0.0$ for the eight elements between $\mathrm{Na}$ and $\mathrm{Ni}$ with suitable lines on the single echellogram. For the heavy elements - $\mathrm{Y}, \mathrm{Zr}, \mathrm{Ba}, \mathrm{La}, \mathrm{Ce}, \mathrm{Pr}$ and $\mathrm{Nd}=[\mathrm{s} / \mathrm{Fe}]$ $\sim-1.2$ with significant scatter which is plausibly attributed to the small number of available lines - a total of 12 distributed over the 7 elements. Luck noted that W Virginis stars show a similar anomaly according to three published analyses (Rodgers and Bell 1963; Barker et al. 1971; Anderson and Kraft 1971). RV Tauri stars lie on an extension of the period-luminosity relation followed by the W Virginis stars. These stars may be on blue loops from the AGB following a He-shell flash. I note that RR Lyrae stars apparently show a similar overdeficiency of the heavy elements over a wide range of metallicities $(-2.2<[\mathrm{Fe} / \mathrm{H}]<0.2)$ - see Butler $(1975)$. Unevolved and other evolved stars with $[\mathrm{Fe} / \mathrm{H}] \sim-1$ do not show an overdeficiency of the heavy elements: the several published analyses show that $[\mathrm{s} / \mathrm{Fe}] \sim 0$ for $[\mathrm{Fe} / \mathrm{H}] \approx-1.5$ with the overdeficiency in more metal-poor stars reaching $[\mathrm{s} / \mathrm{Fe}] \sim-1$ at about $[\mathrm{Fe} / \mathrm{H}]=-2.5$. The over-deficiency of the heavy elements at $[\mathrm{Fe} / \mathrm{H}] \sim-1$ sets $\mathrm{RSct}, \mathrm{W}$ Vir and RR Lyr stars apart from normal stars. Luck suggested that the anomalous combination of $[\mathrm{Fe} / \mathrm{H}]$ and $[\mathrm{s} / \mathrm{Fe}]$ arises because the atmospheres are hydrogen poor thanks to the ravages of stellar evolution: the [s/Fe] for R Sct implies [Fe/H] $\sim-2.5$ at birth and a current hydrogen deficiency of a factor of 30. The hydrogen converted to helium gives a He mass fraction $\mathrm{Y} \sim 0.99$. Direct determination of the He content is difficult. I shall not catalog additional arguments supporting or challenging Luck's inference. The acid test should come, as Luck noted, from analyses of these variable stars in globular clusters where the metallicities of variable stars and "normal" red giants may be compared.

The perception that hydrogen deficiency is a common characteristic of peculiar giants appears to be contagious! Campbell (1985) in an imaginative paper on the origin of the super-metal rich stars proposes that they result from the coalescence of stars in a binary system. The envelope of the resulting single star is expected to be helium-rich. Fortunately, Campbell describes a couple of spectroscopic signatures that such a star should possess; e.g., an enhanced ${ }^{17} \mathrm{O}$ abundance. A search for these signatures will test the proposal.

Other peculiar red giants have been shown recently not to be hydrogen deficient. Sneden and Pilachowski (1984) exploiting precepts outlined by Böhm-Vitense (1979) combined observations of $\mathrm{CO}$ and $\mathrm{CH}$ lines in weak $\mathrm{G}$-band $\mathrm{K}$ giants to show that the atmospheres are hydrogen-rich $(\mathrm{Y}<0.3)$. Böhm-Vitense $(1979)$ shows that $\mathrm{H}$ deficiency and $\mathrm{He}$ enrichment influences the pressures and, hence, the partial pressure of neutral atoms of low ionization potential. Dominy (1984) in an analysis of warm (early-R type) carbon giants used $\mathrm{Ca}$ I lines to show that their atmospheres are hydrogen-rich, i.e., the derived $\mathrm{Ca} / \mathrm{Fe}$ ratio was solar, as expected, provided that the $\mathrm{He} / \mathrm{H}$ ratio was approximately normal.

In 'A Prefatory Observation on Modern Biography', Samuel Taylor Coleridge, the English essayist and poet, wrote "How mean a thing a mere fact is except as seen in the 
light of some comprehensive truth" (Coleridge 1810). To the biographer of the stars, the chemical composition of the stellar atmosphere is a mere fact to be seen in the light of stellar evolution and nucleosynthesis. Both mere facts and comprehensive truth are the biographer's responsibility. I stumbled across Coleridge's dictum in a critical biography of A. E. Housman, the English classical scholar and poet, where the author remarks "there are times when a comprehensive truth must defer to mere fact" (Page 1983). Today, our knowledge of the mere facts about the cool hydrogen deficient stars is so incomplete that we can only guess at the comprehensive truth. While biographers of poets as reticent as Housman may be permanently inhibited in their search for the comprehensive truth by a lack of mere facts, we can assemble a full set of the mere facts about cool hydrogen deficient stars by observing them with telescopes and spectrometers. I hope that the next reviewer of these rare stars may be able to shed more light on 'the comprehensive truth'.

I thank several colleagues for helpful discussions: Dr. P. L. Cottrell, C. Sneden, V. V. Smith, and J. Tomkin. I am especially grateful to Drs. K. Eriksson, B. Gustafsson, and K. H. Hinkle for their essential and substantial contributions to the analyses of the carbon stars which form the basis for $\$ 4$. This research has been supported in part by the National Science Foundation (grant AST 83-16635) and the Robert A. Welch Foundation.

\section{REFERENCES}

Aller, L. H., and Czyzak, S. J. 1983, Ap. J. Suppl., 51, 211.

Anderson, K. S., and Kraft, R. P. 1971, Ap. J., 167, 119.

Barker, T., Baumgart, L. D., Butler, D., Cudworth, K. M., Kemper, E., Kraft, R. P., Lorre, J., Rao, N. K., Reagan, G. H., and Soderblom, D. R. 1971, Ap. J., $165,67$.

Berman, L. 1935, Ap. J., 81, 369.

Bidelman, W. P. 1953, Ap. J., 117, 25.

Böhm-Vitense, E. 1979, Ap.J., 234, 521.

Bond, H. E., Luck, R. E., and Newman, M. J. 1979, Ap. J., 233, 205 (BLN).

Butler, D. 1975, Ap. J., 200, 68.

Cameron, A. G. W., and Fowler, W. A. 1971, Ap. J., 164, 111.

Campbell, B. 1985, preprint.

Canal, R., Isern, J., and Sanahuja, B. 1977, Ap. J., 214, 189.

Clegg, R. E. S., Lambert, D. L., and Tomkin, J. 1981, Ap. J., 250, 262.

Climenhaga, J. L. 1960, Pub. D. A. O. Vic., 11, 307.

Coleridge, S. T. 1810, The Friend, No. 21.

Cottrell, P. L., and Lambert, D. L. 1982a, Ap. J., 261, 595 (CL). 1982b, Observatory, 102, 149.

Danziger, I. J. 1965, M.N.R.A.S., 130, 199 (D65).

Deupree, R. G., and Cole, P. W. 1981, Ap. J. (Letters), 249, L35.

Dominy, J. F. 1984, Ap. J. Suppl., 55, 27.

Dominy, J. F., Wallerstein, G., and Suntzeff, N. B. 1986, Ap. J., in press.

Dufour, R. J., Shields, G. A., and Talbot, R. J., Jr., 1982, Ap. J., 252, 461.

Eriksson, K., Gustafsson, B., Jørgensen, U. G., and Nordlund, § 1984, Astr. Ap., $132,37$.

Fowler, W. A., Caughlan, G. R., and Zimmerman, B. A. 1975, Ann. Rev. Astr. Ap., 13,69 .

François, P. 1985, Astr. Ap., submitted. 
Goebel, J. H., and Johnson, H. R. 1984, Ap. J. (Letters), 284, L39.

Goorvitch, D., Goebel, J. H., and Augason, G. C., Ap. J., 240, 588.

Grevesse, N. 1984, Phys., Scr., T8, 49 (G84).

Härm, R., and Schwarzschild, M. 1975, Ap. J., 200, 234.

Heber, U. 1983, Astr. Ap., 118, 39 (H83).

Heber, U., and Schönberner, D. 1981, Ap. J., 102, 73.

Holweger, H., and Kovács, N. 1984, Astr. Ap., 132, L5.

Hunger, K., Schönberner, D., and Steenbock, W. 1982, Astr. Ap., 107, 93 (HSS).

Iben, I., Jr. 1984, Ap. J., 277, 333.

Iben, I., Jr., and Tutukov, A. V. 1985, Ap. J. Suppl., 58, 661.

Jacoby, G. H., and Ford, H. C. 1983, Ap. J., 266, 298

Johnson, H. R., Goebel, J. H., Goorvitch, D., and Ridgway, S. T. 1983, Ap. J. (Letters), 270, L63.

Johnson, H. R., Alexander, D. R., Bower, C. D., Lemke, D. A., Luttermoser, D. G., Petrakis, J. P., Reinhart, M. D., Welch, K. A., and Goebel, J. H. 1985, Ap. J., 292, 228.

Keenan, P. C., and Greenstein, J. L. 1963, Contr. Perkins Obs. $2,13$.

Kilkenny, D. 1982, M.N.R.A.S., 200, 1019.

Lambert, D. L. 1985, in Cool Stars with Excesses of Heavy Elements, ed. M. Jaschek, and P. C. Keenan (Dordrecht: Reidel), p. 191.

Lambert, D. L., Gustafsson, B., Eriksson, K., and Hinkle, K. H. 1986, Ap. J. Suppl., in press.

Luck, R. E. 1981, P.A.S.P., 93, 211.

Luck, R. E., and Lambert, D. L. 1981, Ap. J., 245, 1018.

Malaney, R. A. 1985, M.N.R.A.S., 216, 743 (M85).

Nissen, P. E. 1983, in Proc. ESO Workship on 'Primordial Helium', ed. P. A. Shaver, D. Kunth, and K. Kjär (Garching: ESO), p. 163.

Nissen, P. E., Edvardsson, B., and Gustafsson, B. 1985, in Proc. ESO Workshop on "Production and Distribution of $C, N, O$ Elements", in press.

Orlov, M. Ya., and Rodriguez, M. H. 1974, Astr. Ap., 31, 203 (OR74). . 1981a, Soviet Astr. Lett., 7, 126 (OR81a).

1981b, Soviet Astr. Lett., 7, 382 (OR81b).

Paczynski, B. 1971, Act. Astr., 21, 1.

Page, N. 1983, in 'A. E. Housman, a critical Biography', (New York: Schocken Books), p. 5.

Querci, M., and Querci, F. 1978, Astr. Ap. 70, L45.

Rao, N. K., Ashok, N. M., and Kulkarni, P. V. 1980, J. Astr. Ap., 1, 71.

Renzini, A. 1979, in Stars and Star Systems , ed. B. E. Westerlund (Dordrecht: Reidel), p. 155.

R. Stalio (Dordrecht: Reidel), p. 319.

Rodgers, A. W., and Bell, R. A. 1963, M.N.R.A.S., 125, 487.

Rufus, W. C. 1915, Pub. Michigan Obs., 2, 103.

Schönberner, D. 1975, Astr. Ap., 44, 381 (S75).

1977, Astr. Ap., 57, 437.

Searle, L. 1961, Ap. J., 133, 531 (S61).

Smith, V. V., and Lambert, D. L. 1985, Ap. J., 294, 326.

1986, Ap. J., submitted.

Sneden, C., and Pilachowski, C. A. 1984, P.A.S.P., 96, 38.

Tomkin, J., Balachandran, S., and Lambert, D. L. 1985, Ap. J., 290, 289.

Tomkin, J., and Lambert, D. L. 1983, Ap. J., 273, 722. 
Tsuji, T.1981, J. Astr. Ap., 2, 95. . 1983, Astr. Ap., 122, 314.

Vardya, M. S. 1966, Observatory, 86, 162.

Warner, B. 1967, M.N.R.A.S., 137, 119.

Zirin, H. 1982, Ap. J., 260, 255. 


\section{DISCUSSION}

GARRISON: When you presented the table with the $\mathrm{C} / \mathrm{He}$ values of the $\mathrm{R}$ CrB stars you said that helium lines are measurable, but you did not quote the values. What were the values?

LAMBERT: If you believe that the helium $\mathrm{D}_{3}$ line is a simple photospheric line, then the numbers are very close to the lower limits.

WING: Would either you or Dr. Liebert care to add a comment about the low-luminosity carbon star G77-61?

LIEBERT: G77-61 is the only star I am aware of with carbon greater than oxygen that is firmly tied to the lower main sequence $\left(\mathrm{M}_{\mathrm{v}}=+11\right)$. It is analyzed in a forthcoming paper (Dearborn, Liebert et $\mathrm{vl}$., 1986 $\mathrm{Jan}$. I. Astrophys. J.). In addition to the near uniqueness of the $\mathrm{C} / 0$ ratio, it is shown in a forthcoming $\mathrm{Ph} . \mathrm{D}$. thesis by $\mathrm{H}$. Gass at Heidelberg (working with $\mathrm{R}$. Wehrse) to be one of the most metal-poor stars known, with $[\mathrm{Fe} / \mathrm{H}]=-3$. There is no evidence for $\mathrm{H}$ deficiency. Dearborn et al. show that G77-61 is a single line spectroscopic binary with a period of 245 days. The most likely evolutionary history invokes mass transfer when the up to now undetected (presumably cool white dwarf) companion transferred a modest amount of $\mathrm{C}$-rich material, probably near maximum radius (AGB phase). The extreme metal deficiency makes it easier to invert the $\mathrm{C} / \mathrm{O}$ ratio, with a minimal amount of mass transfer. This object is obviously in the halo. The kinematics warrants and should receive little more investigation. Perhaps this phenomenon might turn out to be rather less freakish than one thought and the lower main sequences of Omega Centauri and so on might be some of the things accessible.

VARDYA: Is this the star which Greenstein discovered about 1968 ?

LIEBERT: No. I believe this refers to a paper by Greenstein and Oinas, referring to one or two $\mathrm{G}$ dwarfs with enhanced $\mathrm{CH}$ and carbon features and, as I recall, somewhat abnormally high carbon abundances. There are other possible cases - e.g. GH7-21 (Liebert, 1976 AP. J. Letters) - which may relate to subgiant $\mathrm{CH}$ stars (Sneden and Bond), which are too low in luminosity to be explained by post-helium evolution of a single star. G77-61 at $M_{y}=+11$ may just be the lowest luminosity known "CH Star" whose evolution requires a binary mass transfer scenario. (Greenstein is a co-author of our paper, Dearborn et al., 1986, Ap. J.).

FEAST: I was a little surprised that the carbon stars are going to be planetary nebulae. Do we really know enough about the order of evolution and where the carbon stars stand in relation to planetary nebulae. You obviously think so.

LAMBERT: There are some genuinely carbon rich planetary nebulae; for example, they have IR excesses that demonstrate that they contain graphite. So they must come from somewhere. They must come from carbon rich photospheres or envelopes.

FEAST: This is all very nice, but I would not trust it, I even would doubt it. 
LAMBERT: There are two points to that argument. One is you may wish the planetary nebula to be carbon rich because the oxygen has disappeared in the nebula. The other point is that the metal contents of carbon stars are consistent with kinematics when you analyse with normal hydrogen atmospheres. If you make the atmosphere helium rich you loose that consistency. If you are worried about planetary nebulae then I will ask you to take the second point. 